\title{
Effect of ultra-high temperature processing on quality of watermelon
}

\section{juice}

\author{
C. Zhang, Y. Wang, Y. Ma, X. Zhao \& W. Li
}

Beijing Vegetable Research Center, Beijing Academy of Agriculture and Forestry Sciences; Beijing

Key Laboratory of Fruits and Vegetable Storage and Processing; Key Laboratory of Biology and

Genetic Improvement of Horticultural Crops (North China), Ministry of Agriculture; Key Laboratory of

Urban Agriculture (North), Ministry of Agriculture, 9 Shuguanghuayuan Road, Haidian District,

Beijing, China

KEYWORD: Ultra-high temperature; watermelon juice; flavor; quality; GC-MS

ABSTRACT: The effect of ultra-high temperature processing on the quality and flavor of watermelon juice was evaluated. The temperature of 120 and $135 \mathrm{oC}$ was effective to reduce the total flora count to $2 \mathrm{LogCFU} / \mathrm{ml}$ and reduce the color variety of the watermelon juice. However, the higher temperature leaded to a flavor change compared with the un-treatment juice. The ultra-high temperature processing of $120 \mathrm{oC}$ was a proper temperature for the processing of the watermelon juice.

\section{INTRODUCTION}

Watermelon is welcomed all over the world especially in summer. Consumers enjoy the flavor and crisp mouth-feel of the watermelon. Most watermelons are fresh consumed due to the flavor of the watermelon is hard to hold (Zhang, et al., 2010). The thermal pasteurization, exposure to air, contact with the mental vessel and some other exogenous factors all result in the deterioration of the watermelon flavor (Aguiló-Aguayo, et al., 2010, Feng, et al., 2013, Mosqueda-Melgar, et al., 2008).

The technologies, such as the high-intensity pulsed electric field (Oms-Oliu, et al., 2009) , thermosonication (Rawson, et al.), high pressure carbon dioxide (Liu, et al., 2012), and high pressure (Zhang, et al., 2010), have been applied for the processing of the watermelon juice. Among the technologies, ultra-high temperature (UHT) processing gives the high temperature to products in a very short period, and avoid the air exposure of the products, which could be an option for the processing of watermelon juice. The UHT processing has been widely applied in the production of milk and milk-related products (Bönisch, et al., 2004, Lavigne, et al., 1989, Poliseli-Scopel, et al., 2012). However, application of the UHT on the quality and flavor of watermelon was not found to our knowledge.

The influence of the temperature $\left(110,120\right.$, and $\left.135^{\circ} \mathrm{C}\right)$ on the quality of watermelon juice was evaluated. The flavor of the treated juice was compared by the electrical nose.

\section{Material and Methods}

\section{Preparation of watermelon juice}

Watermelons were brought from a local farmer market, which was fresh and mature. The fruits were peeled and put into in a Philips juicer (HR1861, Philips Co. Ltd., Beijing, China). The watermelon juice was mix with a complex food additive that was optimized in our lab. The complex food additive included the carboxymethylcellulose sodium, ascorbic acid, xanthan gum, ethylene diamine tetraacetic acid, carminum, sodium pyrophosphate and etc.. The juice was stirred and adjusted to the $\mathrm{pH} 4.10$ with the citric acid, and adjusted to the soluble solid content (SSC) to 8.0 
Brix $^{0}$ with the high fructose corn syrup (4502504-01, Fresh Juice Industry (Kunshan) Co. Ltd., China). And then the juice was pasteurized by a pilot scale tubular UHT heat exchanger (Model No. FT74 UHT/HTST Processing System, Armfield Technical Education Co. Ltd., Ringwood, UK) at 110,120 and $135{ }^{\circ} \mathrm{C}$ for $15 \mathrm{~s}$, respectively. The outlet temperature was lower than $45{ }^{\circ} \mathrm{C}$. The pasteurized juice was sterile filling in $500 \mathrm{ml}$ PET bottles followed by further cooling in the cold water. The products were store at $4{ }^{\circ} \mathrm{C}$ for the further analysis.

\section{Total flora counts}

Samples were serially diluted, plated in total count agar for total flora counts, followed the recently reported method (Rivasa, et al., 2006). The plates were incubated at $37^{\circ} \mathrm{C}$ for $48 \mathrm{~h}$ and counted manually.

\section{Determination of color and SSC}

Color of the sample was conducted randomly in the reflectance mode for 6 times at $23^{\circ} \mathrm{C}$ (Chromameter CR-300, Minolta, Japan). Color $L^{*}, a^{*}$, and $b^{*}$ value of sample was measured and total color difference $(\Delta \mathrm{E})$ was calculated by Equation $(1)$.

$$
\Delta E=\sqrt{\left(L^{*}-L_{0} *\right)^{2}+\left(a^{*}-a_{0} *\right)^{2}+\left(b^{*}-b_{0}\right)^{2}} \quad(1)
$$

where $\Delta E$ is the total color difference between a sample and the control; $L^{*}, a^{*}$, and $b^{*}$ are the lightness, redness and yellowness of a sample, respectively; $L_{0}, a_{0}$ and $b_{0}$ are the lightness, redness and yellowness of the control.

The SSC of the watermelon juice was recorded by a pocket refractometer (Pal- $\alpha$, ATAGO, Japan) with distilled water as a blank.

\section{Flavor comparison}

The flavor of the samples was compared by the electronic nose PEN2 (Airsense Analytics GmbH, Schwerin, Germany). The electronic nose was turned on for $30 \mathrm{~min}$ and flushed the testing system for $180 \mathrm{~s}$. The sample of $2 \mathrm{ml}$ was put in the testing tube. And then the electronic sensor was put into the testing tube to collect the results for $60 \mathrm{~s}$. The response of the sensor in $48 \sim 52 \mathrm{~s}$ were evaluated by a principal component analysis.

\section{Statistical Analysis}

Analysis of variance (ANOVA) was used to compare mean differences of the results. If the differences in mean existed, multiple comparisons were performed using Duncan's Multiple Range Test. All analysis was conducted using SPSS for Window Version 19. All experiments were done in triplicates or more.

\section{Results and Discussion}

\section{Effect of temperature on total flora count of watermelon juice}

The effect of the UHT temperature on the total flora count of the watermelon juice is shown in Figure 1. The total flora count of the UHT120 and UHT135 was below than $2.0 \mathrm{LogCFU} / \mathrm{ml}$ that was the flora threshold for juice in China, while the total flora count of the UHT110 was higher than 2.0 LogCFU/ml. Moreover, the total flora count of the UHT120 and UHT135 was significantly lower than that of the UHT110. Consequently, the UHT120 and UHT135 accorded with the microbiological criteria of China. 


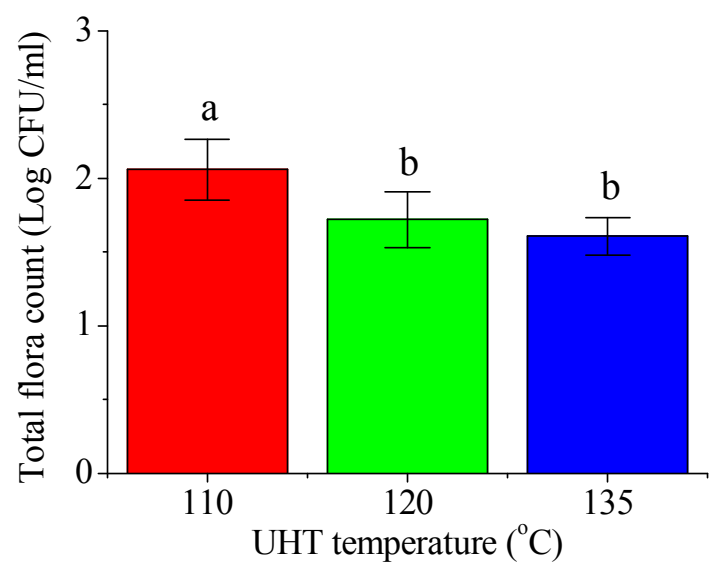

Figure 1 Effect of temperature on the total flora count of watermelon juice

\section{Effect of temperature on quality of watermelon juice}

The effect of UHT temperature on color varieties and SSC of the watermelon juice is shown in Figure 2. The color variety of the 2 samples is hard to be distinguished by normal human vision when the $\Delta E$ between the 2 samples is below 6.0 (Wu \& Sun, 2013). The $\Delta E$ of the UHT110, UHT120 and UHT135 was lower than 6.0 Consequently, the color of the UHT110, UHT120 and UHT135 was not changed significantly. Remarkably, the $\Delta E$ of the UHT120 and UHT135 was significantly lower than that of the UHT110. Being different to our results, the UHT processing of 135 and $140{ }^{\circ} \mathrm{C}$ leads to a significant color variety on the sugarcane juice (Jittanit, et al., 2011). This phenomenon could resulted from the different SSC of the sugarcane juice and watermelon juice. The high temperature could result in the Maillard or caramelization (Bozkurta, et al., 1999, Göğüss, et al., 1998) . The SSC of the watermelon juice was about 8.0 Brix ${ }^{\circ}$, while that of the sugarcane juice was higher than 30 Brix $^{\circ}$. The Maillard or caramelization had more chance to begin in the sugarcane juice.

The UHT temperature showed no influence on the SSC of the watermelon juice. Being similar to our results, the UHT treatment of 135 and $140{ }^{\circ} \mathrm{C}$ shows no influence on the SSC of the sugarcane juice (Jittanit, et al., 2011).

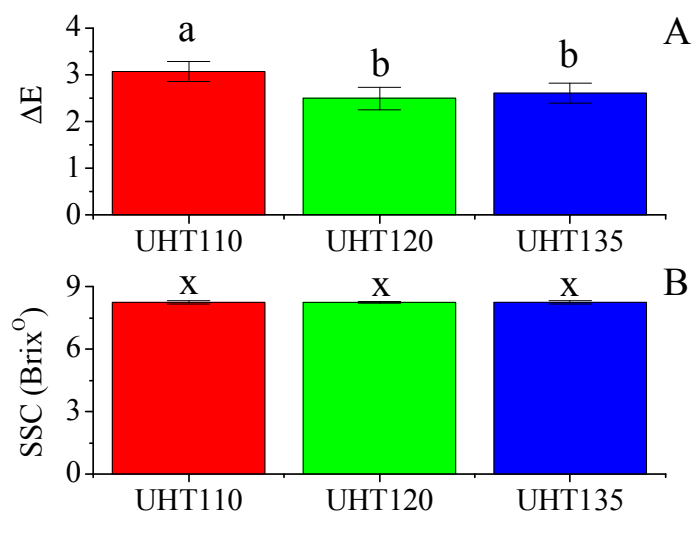

Figure 2 Effect of temperature on the quality of the watermelon juice

\section{Effect of temperature on flavor of watermelon juice}

The flavor of the UHT processed juice was compared with the control juice by the electric nose analysis. The main component 1 and main component 2 contributed $95.32 \%$ and $3.27 \%$ for the flavor of the watermelon juice (Figure 3). Hence, the main component 1 and 2 accounted 98.59\% of the total flavor. The distant of the UHT110 and UHT120 to the control was similar, while that of the UHT135 to the control was longer. Consequently, the flavor of the UHT110 and UHT120 was more similar to that of the control. The higher temperature leaded to the flavor variety of the watermelon juice. 


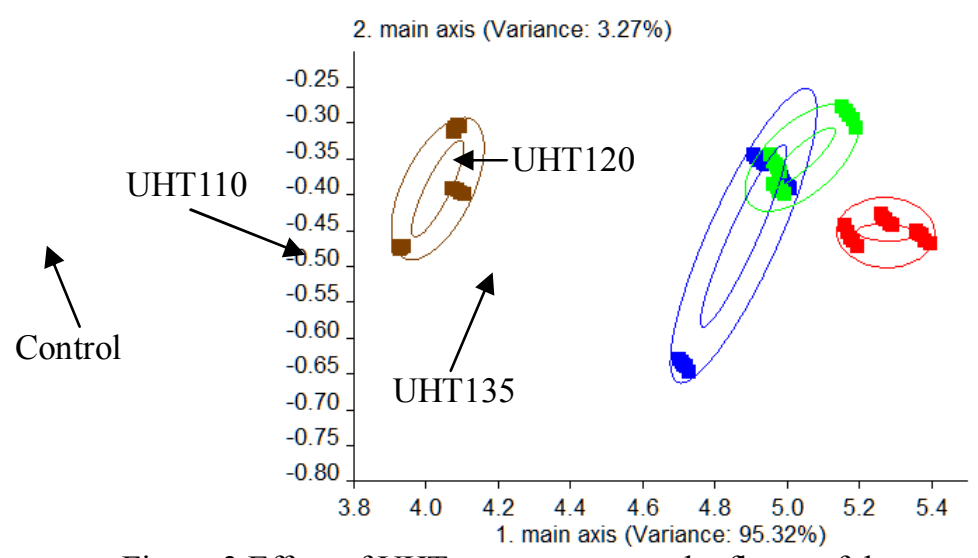

Figure 3 Effect of UHT temperature on the flavor of the watermelon juice

\section{Conclusion}

The effect of ultra-high temperature treatment on the quality and flavor of watermelon juice was evaluated. The temperature of 120 and $135{ }^{\circ} \mathrm{C}$ was effective to reduce the total flora count to 2 $\mathrm{LogCFU} / \mathrm{ml}$ and reduce the color variety of the watermelon juice. However, the high temperature leaded to a flavor change compared with the un-treatment juice. The ultra-high temperature treatment of $120{ }^{\circ} \mathrm{C}$ was a proper temperature for the processing of the watermelon juice.

\section{Acknowledgements}

The authors are grateful to financial support of the earmarked fund for Modern Agro-industry Technology Research System (CARS-26-22 \& CARS-25), Beijing Academy of Agricultural and Forestry Sciences, New Discipline Breeding (KJCX20140204), and Beijing Key Laboratory of Fruits and Vegetable Storage and Processing (Z141105004414037).

\section{References}

[1] Aguiló-Aguayo, I., Montero-Calderón, M., Soliva-Fortuny, R. \& Martín-Belloso, O. 2010. Changes on flavor compounds throughout cold storage of watermelon juice processed by high-intensity pulsed electric fields or heat. Journal of Food Engineering 100(1):43-49.

[2] Bönisch, M.P., Lauber, S. \& Kulozik, U. 2004. Effect of ultra-high temperature treatment on the enzymatic cross-linking of micellar casein and sodium caseinate by transglutaminase. Journal of Food Science 69(8):E398-E404.

[3] Bozkurta, H., Göğüşa, F. \& Eren, S. 1999. Nonenzymic browning reactions in boiled grape juice and its models during storage. Food Chemistry 64(1):89-93.

[4] Feng, M., Ghafoor, K., Seo, B., Yang, K. \& Park, J. 2013. Effects of ultraviolet-C treatment in Teflon ${ }^{\circledR}$-coil on microbial populations and physico-chemical characteristics of watermelon juice. Innovative Food Science and Emerging Technologies 19:133-139.

[5] Gögüsş, F., Bozkurt, H. \& Eren, S. 1998. Kinetics of maillard reactions between the major sugars and amino acids of boiled grape juice. LWT 31(2):196-200.

[6] Jittanit, W., Wiriyaputtipong, S., Charoenpornworanam, H. \& Songsermpong, S. 2011. Effects of varieties, heat pretreatment and UHT conditions on the sugarcane juice quality. Chiang Mai Journal of Science 38(1):116-125.

[7] Lavigne, C., Zee, J.A., Simard, R.E. \& Béliveau, B. 1989. Effect of processing and Sstorage conditions on the fate of vitamins B1, B2, and C and on the shelf-life of goat's milk. Journal of Food Science 51(1):30-34.

[8] Liu, Y., Hu, X., Zhao, X. \& Song, H. 2012. Combined effect of high pressure carbon dioxide and mild heat treatment on overall quality parameters of watermelon juice. Innovative Food Science and Emerging Technologies 13:112-119. 
[9] Mosqueda-Melgar, J., Raybaudi-Massilia, R.M. \& Martin-Belloso, O. 2008. Combination of high-intensity pulsed electric fields with natural antimicrobials to inactivate pathogenic microorganisms and extend the shelf-life of melon and watermelon juices. Food Microbiology 25(3):479-491.

[10] Oms-Oliu, G., Odriozola-Serrano, I., Soliva-Fortuny, R. \& Martin-Belloso, O. 2009. Use of Weibull distribution for describing kinetics of antioxidant potential changes in fresh-cut watermelon. Journal of Food Engineering 95(1):99-105.

[11] Poliseli-Scopel, H.F., Hernández-Herrero, M., Guamis, B. \& Ferragut, V. 2012. Comparison of ultra high pressure homogenization and conventional thermal treatments on the microbiological, physical and chemical quality of soymilk. LWT 46(1):42-48.

[12] Rawson, A., Tiwari, B.K., Patras, A., Brunton, N., Brennan, C., Cullen, P.J. \& O'Donnell, C. Effect of thermosonication on bioactive compounds in watermelon juice. Food Research International 44(5):1168-1173.

[13]Rivasa, A., Rodrigoa, D., Martíneza, A., Barbosa-Cánovasb, G.V. \& Rodrigo, M. 2006. Effect of PEF and heat pasteurization on the physical-chemical characteristics of blended orange and carrot juice. LWT 39(10):1163-1170.

[14] Wu, D. \& Sun, D.-W. 2013. Colour measurements by computer vision for food quality control - A review. Trends in Food Science \& Technology 29(1):5-20.

[15]Zhang, C., Trierweiler, B., Li, W., Butz, P., Xu, Y., Rufer, C.E., Ma, Y. \& Zhao, X. 2010. Comparison of thermal, ultraviolet-c, and high pressure treatments on quality parameters of watermelon juice. Food Chemistry 126(1):254-260. 